\title{
FAINT STRUCTURES IN LOW DENSITY REGIONS OF THE NEARBY UNIVERSE
}

\author{
U. LINDNER AND K.J. FRICKE \\ Universitäts-Sternwarte, Göttingen, Germany \\ AND
}

J. EINASTO AND M. EINASTO

Tartu Astrophysical Observatory, Töravere, Estonia

We present an investigation of the galaxy distribution in the huge underdense region between the Hercules, Coma and Local Superclusters, the so-called Northern Local Void (NLV), using void statistics (for details refer to Lindner et al. this Volume). Reshift data for galaxies and poor clusters of galaxies are available in low and high density regions as well. Samples of galaxies with different morphological type and various luminosity limits have been studied separately and void catalogues have been compiled from three different luminosity limited galaxy samples for the first time. Voids have been found using the empty sphere method which has the potential to detect and describe subtle structures in the galaxy distribution. Our approach is complementary to most other methods usually used in Large-Scale Structure studies.

We found that the resulting sets of voids form a hierarchical system: The fainter the limiting luminosity of the galaxies the smaller are the voids defined by them. Voids outlined by bright galaxies are interlaced by a fine network of faint galaxy filaments dividing them into smaller subvoids. Considering the same type of surrounding objects, mean void diameters are larger in low density regions than in supercluster regions indicating that in low density regions not only systems of galaxies are poorer (this follows from the definition of low density regions) but also systems of galaxies are spaced more widely. This Void Hierarchy is an important property of the Large-Scale Structure in the Universe which constrains any realistic galaxy and structure formation scenario. In addition, this concept of Void Hierarchy may help to devise new concepts for the study of the Large-Scale Structure in the Universe. 